\title{
Intrusive memories of trauma in PTSD and addiction
}

\author{
Martina Reynolds, ${ }^{1}$ Sheena Nayak, ${ }^{1}$ Christos Kouimtsidis ${ }^{2}$
}

The Psychiatrist (2012), 36, 284-289, doi: 10.1192/pb.bp.111.037937

${ }^{1}$ School of Social Sciences, Brunel University, London, UK; ${ }^{2}$ West Herts Community Drug and Alcohol Team, Hertfordshire Partnership NHS Foundation Trust, UK

Correspondence to Christos Kouimtsidis

(drckouimtsidis@hotmail.com)

First received 21 Nov 2011, final revision 23 Jan 2012, accepted 29 Feb 2012

\begin{abstract}
Aims and method To study intrusive phenomena relating to traumatic experiences in a community sample seeking treatment for substance use disorder and an inpatient sample from an addiction in-patient detoxification service in London. Perceived effect of drugs and drug use on traumatic intrusion and memory experiences was also analysed. The study was conducted using a semi-structured patient interview comprising a series of questionnaires.
\end{abstract}

Results The most frequently identified traumatic memories were those of traumatic bereavements. Substance use appears to have had the effect of dampening the memories for those with post-traumatic stress disorder (PTSD), and significantly more individuals reported that their memories were less vivid and less distressing before they ceased substance use.

Clinical implications The findings suggest that continued substance use could in part be motivated by its capacity to dampen distressing memories within the PTSD group. This should be addressed within a treatment plan of comorbid PTSD.

Declaration of interest None.
The connection between trauma, post-traumatic stress disorder (PTSD) and substance use has been well documented. It seems that many, but not all individuals who use substances have a history of trauma or ongoing traumatic events in their lives. ${ }^{1,2}$ Many individuals who use substances lead lives that involve a lot of risks, making them vulnerable to trauma. ${ }^{1,3-5}$ Therefore, trauma can be a predisposing factor or the result of substance misuse. Despite some of the precarious traumas recounted, many people adapt and do not seem to be severely psychologically affected by the risky lifestyle. ${ }^{6}$ What is perhaps more poignant is the types of traumas that those who use substances are affected by: deaths of close family, loved ones and traumatic bereavements were identified as most traumatic by a significant percentage of participants, both in terms of experience and memories that they continued to be troubled by. ${ }^{6,7}$ It seems that despite substance use and the accompanying risky lifestyle some individuals manage to maintain a satisfactory level of psychological and general well-being, ${ }^{6}$ yet there are also those on whom this lifestyle takes its toll, and traumatic experiences manifest as disturbing symptoms that continue to trouble them. Substance use becomes a comfort or means of escape from an otherwise disturbed psyche in line with ego psychology and object relations theories. ${ }^{8}$

\section{Research background}

The complicated picture of symptom overlap between trauma, PTSD and substance use has also been documented.
Disturbing psychological symptoms prevalent in PTSD are common in substance misuse, for example depressed mood, anxiety, irritability, physiological arousal, hypervigilance and impaired social and occupational functioning. There are also substance intoxication and withdrawal symptoms that are similar to PTSD symptoms such as impairment in attention or memory, sleep disturbance, fatigue, vivid unpleasant dreams and intrusive images and flashbacks. Some substance-related effects may obscure PTSD symptomatology, for instance euphoric and relaxing effects of opiates may provide relief from depression and anxiety associated with PTSD. Blunted or flattened affects occur in PTSD, but can also be characteristic of the toxic effects of cannabis or heroin. ${ }^{9}$

Several early studies were based on the self-medication hypothesis, and the overall conclusion supports the notion that substance use was associated with self-management of symptoms such as anxiety, depression, inability to cope with emotions, low self-esteem, attention-deficit problems and interpersonal relationship problems. ${ }^{10,11}$ Conclusions were based on clinical observation.

Khantzian ${ }^{12,13}$ hypothesised that there was a relationship between the individual, the drug and its effects on the individual's mental state. People with heroin dependence preferred the calming and dampening effects of opiates. They used them to counter feelings of rage, shame and loneliness, and it reinforced their tendency to isolation and withdrawal. ${ }^{14,15}$ In contrast, those who misused amphetamine favoured its stimulating effects that enhanced 
self-worth and a defensive style that provoked embracing confrontation with their environment. More recently, functional associations between PTSD and substance use disorders have been explored by investigating effects of different drugs on different PTSD symptom categories (intrusion, avoidance and arousal). McFall et $a l,{ }^{16}$ studying a sample of combat-exposed veterans, discovered that alcohol problems were significantly correlated with intrusion and arousal symptoms but not avoidance symptoms, whereas drug problems were significantly correlated with the avoidance/numbing and intrusion symptoms, but not with arousal. These findings were replicated by Stewart et $a l^{9}$ in women with substance misuse who were not seeking treatment recruited from the community. Such findings have implications for effectiveness of treatment. Intoxication, as a form of avoidance and defence, may provide short-term symptom relief, but in the longer term may prevent habituation to traumatic memories due to inadequate exposure for processing, or couched in psychoanalytic terms, facilitate repression or suppression that prevents recovery. ${ }^{9}$

There is a large body of research documenting characteristics of traumatic memories outside the substance misuse field, and, of relevance, several studies identified intrusive memories as being characteristic of depression as well as PTSD bereavements. ${ }^{17-19}$ Reynolds \& Brewin ${ }^{19}$ studied the nature of intrusive memories in a clinical sample, and found in an analysis of intrusive memory content of combined depression and PTSD samples that $93 \%$ of events concerned death, illness or injury to family members, illness or injury to the patient, assault on the patient, and interpersonal problems. Such traumas and many others are also common in the risk-taking lifestyle of those who use substances. ${ }^{6-7}$

This study is part of a larger project exploring the relationship between trauma, PTSD and substance use disorders in UK clinical populations; other aspects of the project are reported by Reynolds et al. ${ }^{6,7}$ The aim of this study is to analyse the nature and characteristics of intrusive phenomena relating to traumatic experiences in a cohort that comprises a community sample seeking treatment for substance use disorders and an in-patient sample from a specialist in-patient addiction service in south London. The perceived effect of drugs and drug use on traumatic intrusion and memory experiences will also be explored.

\section{Method}

The interview schedule and assessments used by Reynolds et $a l^{7}$ were used to facilitate a comparison of the findings. All assessments were completed during the interview which took approximately $1.5 \mathrm{~h}$. Clients were reimbursed for expenses only. Written consent was obtained before participation in the study.

\section{Participants}

The community sample was recruited from clients attending a community drug and alcohol service, which covers a large suburban and rural community outside
London. The in-patient sample was recruited from an addiction in-patient detoxification unit in south London. All clients aged 18-70 years were approached, and only those with current severe mental illness or organic impairment which would affect their ability to participate were excluded. The researchers had no knowledge of clients' histories before interviewing, so all who participated were representative of the community drug and alcohol service and in-patient populations in general.

\section{Assessment}

Demographic details, trauma exposure, PTSD, substance use and associated problems, and other comorbid disorders were assessed using the semi-structured interview schedule and questionnaires as described below.

\section{Addiction Severity Index (ASI) ${ }^{20}$}

An interview to detect and measure severity of problems in seven areas commonly affected by alcohol and drug dependence: ethnic/cultural, medical, employment/support, drug/alcohol use, legal status, family/social relationships and psychiatric. Severity is indicated by a composite score for each area derived from responses to specific questions in each section.

\section{Trauma History Questionnaire (THQ) ${ }^{21}$}

This consists of 24 items addressing experience, number of times and age at occurrence with respect to a range of traumatic events in three areas: crime-related events, general disaster and trauma, and unwanted physical and sexual experiences. Additional ratings included distress at the time of the trauma and currently. After completing the THQ, participants were asked to identify the trauma that they felt was the most distressing and had the worst impact on them overall.

\section{PTSD Symptom Scale (Interview Version) (PSS-I) ${ }^{22}$}

This is a 17-item scale designed to assess PTSD symptomatology and consists of questions pertaining to the symptoms in the re-experiencing, avoidance and arousal categories of PTSD. This interview was completed in relation to the trauma identified as the most distressing overall in the THQ, referred to as the 'target trauma' throughout this paper. A diagnosis of PTSD is made on the basis of meeting the DSM-IV-TR criteria for PTSD. ${ }^{23}$ The items endorsed on the scale can also be summed to give a total score for symptom groups B, C and D of the DSM-IV-TR. A score of 17 or more indicated a cut-off for PTSD.

\section{Brief Symptom Inventory (BSI) ${ }^{24}$}

This is a 53-item self-report symptom inventory which reflects the 9 primary symptom dimensions (somatisation, obsessive-compulsive, interpersonal sensitivity, depression, anxiety, hostility, phobic anxiety, paranoid ideation and psychoticism) of the Symptom Checklist-90-Revised (SCL-90-R) and is designed to assess the psychological symptom status of psychiatric and medical patients. Other symptom measures derived from the questionnaire include global severity index, positive symptom total and positive symptom distress. Internal consistency $\alpha=0.71-0.85$. 


\section{Social functioning}

Social functioning was assessed by asking participants to rate on a visual analogue scale, in which $0=$ not at all and $10=$ very much so, how much the trauma-associated problems had interfered with their work, social life/leisure activities and family life/home responsibilities.

\section{Traumatic memory and trauma characteristics interview} This interview is based on Reynolds \& Brewin $^{19}$ and is discussed in Reynolds et $a l^{7}$ Clients were asked the following questions: (i) describe the memory of trauma identified as the worst trauma; (ii) age at time of the trauma; (iii) intoxication at the time of the trauma (answers were coded: $0=$ no, $1=$ yes); (iv) drug use before and after the trauma; (v) whether drug use increased, decreased or remained the same after the trauma; (vi) whether the quality of the memories changed since drug use stopped ( $1=$ less vivid before drug use stopped, $2=$ more vivid before drug use stopped, $3=$ the same); (vii) whether the memories came to mind before drug use stopped ( $1=$ hardly at all, $2=$ as frequently as now, $3=$ less frequently, $4=$ more frequently); (viii) how distressing the memory was compared with before drug use stopped $(1=$ more distressing, 2 = less distressing, 3 = about the same); (ix) how distressing the memories were currently (rated on a visual analogue scale in which $0=$ not at all and $10=$ extremely); (x) how distressing the memories were before drug use stopped (rated on a visual analogue scale in which $0=$ not at all and $10=$ extremely); and (xi) whether they thought there was a connection between their drug use and the trauma $(0=$ none, $1=$ escape, blocking it out and coping, $2=$ yes, specific relationship identified, $3=$ yes, but no specific relationship identified).

\section{Case-note review}

A case-note review was undertaken and the following information was obtained: (i) whether reference was made to any past traumas in the case notes (for the purpose of this study, reference to items listed in the THQ was used as a guide for definition of a trauma; answers were coded yes/ no); (ii) nature of traumas documented; (iii) number of traumas documented; (iv) whether a PTSD diagnosis was made/referred to (answers were coded yes/no). If PTSD diagnosis was made, was the patient referred for treatment? Answers were coded yes/no. The notes were screened for all traumas documented.

\section{Results}

\section{Data analysis}

Demographic data and qualitative characteristics of the sample are described. Analysis was carried out for differences between groups (PTSD $v$. non-PTSD) in all assessments and measures. Comparisons between groups are made using parametric statistics ( $t$-tests) and equivalent non-parametric statistics where appropriate.

Overall, 92 clients were included. The mean age of the sample was 35.6 years $($ s.d. $=8.5)$ and there was no significant difference between in-patients and out-patients $(t=-0.99$, d.f. $=88, P>0.10)$ or males and females $(t=0.53$, d.f. $=88, P>0.10)$. Sixty-seven participants $(72.8 \%)$ had a qualification, skill or profession. For more details about demographic data see Table 1.

\section{Trauma and PTSD}

There were 31 participants (33.7\%) who met DSM-IV-TR criteria for PTSD at the time of the interview, 8 (8.7\%) were categorised as having a partial PTSD, and $53(57.6 \%)$ did not meet the PTSD criteria. Further, 81 participants (88\%) reported two or more DSM-IV-TR criterion A events for PTSD, 9 (9.8\%) reported one event, and $2(2.2 \%)$ did not report any. Of target traumas reported, 78 (84.8\%) met PTSD criterion A definition, 8 (8.7\%) were borderline and 6 (6.5\%) did not meet the criterion specification. All traumas reported by the PTSD group met criterion A specification. Traumatic experiences were common, and the majority of the participants reported experiencing a range of traumas, irrespective of PTSD diagnosis, and for all trauma categories: mugging, robbery, break-ins, serious accidents, natural or man-made disaster, witnessing serious injury or death, traumatic bereavements, sexual assault and physical violence, threat and assault. The differences between the PTSD and non-PTSD groups were not significant. There was one exception: sexual abuse was reported by significantly more of the PTSD group than the non-PTSD group $\left(\chi^{2}(1)=25.04, \quad P<0.0005\right)$. Traumatic experiences are summarised in Table 2.

Further details concerning trauma and PTSD can be found in Reynolds et al. ${ }^{6,7}$ The subsequent analyses in this report will involve comparisons between the PTSD group and the non-PTSD group. The former includes those who fulfilled the criteria for a DSM-IV-TR diagnosis at the time of the interview, the non-PTSD group therefore includes those who did not meet criteria for the diagnosis and also those who met criteria for a partial diagnosis as described earlier, as in Reynolds et al. ${ }^{7}$ The 'partial PTSD' group were included in the non-PTSD group for the main analysis because on the whole their scores were within the range of the non-PTSD group.

\begin{tabular}{|lc|}
\hline Table 1 Demographic information on the sample \\
Variable & $n(\%)$ \\
\hline In-patient & $51(55.4)$ \\
\hline Male & $57(62)$ \\
\hline White & $82(89.1)$ \\
\hline Occupation & \\
Unemployed & $51(55.4)$ \\
Employed & $32(34.8)$ \\
Retired/sick & $8(8.7)$ \\
\hline Living arrangements & \\
Never married & $53(57.6)$ \\
Married & $17(18.5)$ \\
Divorced/separated & $22(33.9)$ \\
Alone & $30(32.6)$ \\
Partner only & $18(19.6)$ \\
Partner and children & $17(18.5)$ \\
Parents or family & $8(8.7)$ \\
Children only & 7 \\
Friends & $4(7.6)$ \\
Not stable & $8(8.4)$ \\
\end{tabular}




\begin{tabular}{|c|c|c|c|c|}
\hline Trauma category & $\begin{array}{l}\text { PTSD group, } n \\
\quad(n=31)\end{array}$ & $\begin{array}{l}\text { Non-PTSD group, } n \\
\quad(n=61)\end{array}$ & $\begin{array}{c}\text { Total } \\
(n=92)\end{array}$ & Statistic \\
\hline Mugging, robbery, break-ins & 27 & 47 & 74 & $\chi^{2}(1)=1.32$ \\
\hline Serious accidents, natural or man-made disaster & 23 & 41 & 64 & $\chi^{2}(1)=0.47$ \\
\hline Witnessed serious injury or death & 19 & 42 & 61 & $\chi^{2}(1)=0.53$ \\
\hline Traumatic bereavements & 26 & 48 & 74 & $\chi^{2}(1)=0.35$ \\
\hline Miscellaneous & 15 & 28 & 43 & $\chi^{2}(1)=0.05$ \\
\hline Sexual assault & 22 & 11 & 33 & $\chi^{2}(1)=25.04^{\star \star \star}$ \\
\hline Physical violence, threat, assault & 21 & 39 & 60 & $\chi^{2}(1)=1.31$ \\
\hline
\end{tabular}

PTSD, post-traumatic stress disorder.

${ }^{\star \star \star *} P<0.0005$.

\section{Trauma and drug use}

In total, 31 participants (33.9\%) said that they were intoxicated at the time of the target trauma, and although the difference between the PTSD and non-PTSD groups did not quite reach significance, almost $50 \%$ of the PTSD group reported being intoxicated compared with about $30 \%$ of the non-PTSD group (PTSD 15/31, non-PTSD 18/61; $\chi^{2}(1)=3.18$, $P<0.10)$. For $61(66.3 \%)$ participants, their drug use increased after the target trauma, and for 26 (28.3\%) it decreased or remained the same; the PTSD group did not differ significantly from the non-PTSD group (PTSD 23/ 30, non-PTSD 39/59; $\left.\chi^{2}(1)=1.05, \quad P<0.10\right)$. Fifty-three participants $(57.6 \%)$ felt that there was a connection between their drug use and the trauma. Twenty (21.7\%) described their substance use as a means of escape or blocking, numbing or helping them to cope. Examples include 'helped escape, numbed feeling', 'numbs the memories, helps' and 'to block pain'. Similarly, 20 participants (21.7\%) described another specific connection, examples of which included 'due to paranoia and low selfesteem', 'shock of diagnosis of cancer'; 14 (15.2\%) felt that there was a connection, but not a specific one, and 37 (40.2\%) reported no connection. There was a significant difference between the PTSD and non-PTSD groups (PTSD $26 / 31$, non-PTSD 27/60; $\left.\chi^{2}(1)=12.70, \quad P<0.0005\right)$ as a greater proportion of the PTSD group reported a connection between drug use and the trauma, but of those who did, there was no significant difference between the PTSD and non-PTSD groups in the number who described their substance use as a means of escape/blocking (PTSD 12/26, non-PTSD 8/27; $\left.\chi^{2}(1)=1.54, P>0.10\right)$. Fifty-eight people (63\%) had been using one or more substances before the target trauma, and 31 who had not used any substances before the trauma started using them after the trauma.

\section{Traumatic memory characteristics}

The memories identified by participants were divided into six categories: traumatic bereavements, witnessing serious illness or accident in others, sexual abuse, assault, personal illness, accident or injury and miscellaneous traumas (Table 3). Memories of traumatic bereavements were noteworthy as being identified by 38 participants, 13 in the PTSD group and 25 in the non-PTSD group, amounting to approximately $40 \%$ of each group and the two groups combined. Thirteen participants reported memories of sexual abuse, eight in the PTSD group and five in the non-PTSD group.

When asked how substance consumption affected the quality of their memories, 40 participants said that the memories were less vivid before they ceased substance use, 24 said that they were more vivid, and 23 reported that they were the same. On exploring differences between the PTSD and non-PTSD groups, it was found that there was a significant difference between the groups as significantly more of the PTSD group reported that their memories were less vivid before they ceased substance use (PTSD: less vivid 20/31, more vivid 5/31, the same 6/31; non-PTSD: less vivid $20 / 56$, more vivid $19 / 56$, the same $17 / 56 ; \chi^{2}(2)=6.80$, $P<0.05)$. In response to the question of how distressing they found the memory compared with before they ceased using substances, 29 found it more distressing, 19 less distressing and 38 the same. Further analysis showed that once again the PTSD and non-PTSD groups differed significantly, and 18/31 of the former found the memory more distressing compared with $11 / 55$ of the latter (PTSD: more distressing $18 / 31$, less distressing $7 / 31$, the same $6 / 31$; non-PTSD: more distressing $11 / 55$, less distressing $12 / 55$, the same $\left.32 / 55 ; \chi^{2}(2)=15.28, P<0.0005\right)$.

When asked about the frequency of the memories before substance use stopped, 27 reported that they rarely experienced them, 26 experienced them as frequently as currently, 23 less frequently, and 8 more frequently. The differences between the PTSD and non-PTSD groups were not significant in this instance (PTSD: less frequently 18/30, same or more frequently 12/30; non-PTSD: less frequently

\begin{tabular}{|lcc|}
\hline Table 3 Target memories & $\begin{array}{c}\text { PTSD } \\
\text { group, } n\end{array}$ & $\begin{array}{c}\text { Non-PTSD } \\
\text { group, } n\end{array}$ \\
\hline Memory category & 13 & 25 \\
\hline Traumatic bereavement & 0 & 6 \\
\hline Sexual abuse & 8 & 5 \\
\hline Assault & 6 & 12 \\
\hline Miscellaneous trauma & 2 & 10 \\
\hline Personal illness/injury/accident & 2 & 3 \\
\hline Total & 31 & 61 \\
\hline
\end{tabular}

PTSD, post-traumatic stress disorder. 
$32 / 54$, same or more frequently $22 / 54 ; \quad \chi^{2}(1)=0.004$, $P>0.10)$.

The mean distress rating for memories before using stopped was $5.3($ s.d. $=3.5)$ compared with 5.18 (s.d. $=3.24$ ) since using stopped, so the distress rating decreased when the participants stopped using substances and this difference was significant $(t=14.01$, d.f. $=86, P<0.0005)$. Further exploration of this showed that the distress rating before ceasing substance use was slightly higher for the PTSD group (5.9, s.d. =3.4) than the non-PTSD group (5.0, s.d. =3.6), and the current distress rating was $7.3($ s.d. $=2.4)$ for the PTSD group and 4.0 (s.d.=3.0) for the non-PTSD group, so the distress rating since ceasing substance use increased in the PTSD group, but decreased in the nonPTSD group. It seems that substance use was having the effect of elevating distress associated with the memories for the non-PTSD group, but the opposite was the case for the PTSD group, where distress ratings were elevated following ceasing substance use.

\section{Discussion}

Approximately a third of the sample met criteria for a diagnosis of PTSD. Traumatic events were common, with $88 \%$ reporting two or more DSM-IV-TR criterion A events for PTSD, and $85 \%$ of target traumas reported met PTSD criterion A definition. Therefore, irrespective of PTSD diagnosis, traumatic events were common. This is consistent with previous studies. What appears to differentiate the PTSD and non-PTSD groups is the higher level of psychological distress experienced and the social impact of the trauma on the lives of the former, ${ }^{6,7}$ as expected, given that those with a diagnosis of PTSD by definition have more symptoms and greater impairment than those without the diagnosis.

The most frequently identified traumatic memories were of traumatic bereavements for both the PTSD and non-PTSD groups, despite the high frequency of reported traumatic experiences in other categories. Memories of other traumas were reported less frequently by both groups, including sexual abuse, despite the higher reported rate of this trauma in the PTSD group. Six people in the study sample identified traumatic bereavements involving a drug overdose; others included deaths due to suicide and illness. Most of these individuals also listed other traumatic experiences, some of which could be considered severe traumas, but still identified a traumatic bereavement as being the most traumatic and having the biggest impact on them. This is consistent with findings of previous research with other client groups, showing that the nature of the traumas and target traumatic memories is similar. ${ }^{17-19}$ A lot of individuals with substance use have close encounters with death, through near-overdoses, their lifestyle or the deaths of those around them, so death-related traumas more than any other are a constant reminder on the one hand, and the fantasy of being able to master it on the other, as described by Loose: ${ }^{8}$ 'the junky aesthetic in drug culture is a flirtation with death. It eroticises and provokes death. It derives enjoyment from the image of death' (p. 144).

For the PTSD group, substance use appears to have had the effect of dampening the memories in various ways.
Significantly more participants reported that their memories were less vivid and less distressing before they ceased substance use, although there was no significant difference between the groups with regard to the frequency that memories were experienced before substance use ceased. These findings suggest continued substance use could in part be motivated by its capacity to dampen distressing memories within the PTSD group, therefore for this group, drug use could be understood in terms of self-medication., ${ }^{3,12-15,25}$ This is supported by the high percentage $(57.6 \%)$ that felt that there was a connection between their drug use and the trauma, with $21.7 \%$ describing their substance use as a means of escape or blocking, numbing or helping them to cope. Another 21.7\% described other specific connections relating to coping or otherwise comforting in the face of adversity. The connection between the trauma and drug use was significantly more common in the PTSD group. Fifty-eight participants $(63 \%)$ had been using one or more substances before the target trauma, and 31 people who had not used any substances before the trauma started using after the trauma. This is where understanding and knowledge of the individual's history and attention to such experiences could be helpful in facilitating relapse prevention.

Intoxication at the time of the target trauma was reported by a third of the sample, and there was a tendency for this to be more common in the PTSD group, although the difference was not significant. Substance use increased after the target trauma for approximately two-thirds, independently of the diagnosis of PTSD, suggesting that it is either not specific to PTSD, or that some participants may have had a lifetime diagnosis of PTSD as reported in Reynolds et al. ${ }^{6,7}$

The results from this study need to be interpreted with caution. The sample included patients with substance use disorders attending out-patient and in-patient services for detoxification from substances rather than patients attending PTSD services. Although the out-patient and in-patient samples of the study are not significantly different from each other as far as traumatic experience, substance use, associated psychological and social factors are concerned, ${ }^{6}$ they may not be representative of the overall population attending PTSD, emergency or military support services.

Further study is required to clarify and extend some findings. What can be said is that they are broadly consistent with findings of some other studies exploring characteristics of intrusive memories where people typically try to avoid distressing re-experiencing phenomena characteristic of PTSD. Using substances to achieve this complicates the picture and this is where it takes a slightly different path to other types of avoidance. Therefore, it is important that clinicians involved either with assessment and treatment of people with substance use disorders, or assessment and treatment of people presenting with PTSD, are aware of the risk of comorbidity between substance use disorders and PTSD, the high prevalence of traumatic experiences, and the role of substances used. Intrusive memories are experienced by all who have traumas in their past, but what differentiates those with and without PTSD is the degree of distress associated with them. In this study 
substance use was a form of respite and relief, particularly to those with PTSD for whom the memories of trauma were less vivid before they ceased using substances.

\section{Acknowledgements}

We thank the staff of West Herts Community Drug and Alcohol Team and In-Patient Detoxification Unit of Springfield Hospital for their support and facilitation of this study, as well as the clients who participated and shared their experiences.

\section{About the authors}

Martina Reynolds BA, MA, PhD is a senior lecturer and Sheena Nayak, BSc, MSc is an MSc student, Brunel University, Uxbridge, London; Christos Kouimtsidis, MRCPsych, MSc, PhD is a consultant psychiatrist, West Herts Community Drug and Alcohol Team, Hertfordshire Partnership NHS Foundation Trust, Hemel Hempstead, UK.

\section{References}

1 Kingston S, Raghavan C. The relationship of sexual abuse, early initiation of substance use, and adolescent trauma to PTSD. J Trauma Stress 2009; 22: 65-8.

2 Mills KL, Teesson M, Ross J, Peters L. Trauma, PTSD, and substance use disorders: findings from the Australian National Survey of Mental Health and Well-Being. Am J Psychiatry 2006; 163: 652-8.

3 Wiechelt SA. Trauma and substance misuse: critical considerations in understanding the maelstrom. Subst Use Misuse 2007; 42: 527-33.

4 Darke S, Duflou J. Toxicology and circumstances of death of homicide victims in New South Wales, Australia 1996-2005. J Forensic Sci 2008; 53: 447-51.

5 Howard DE, Qi Wang M. Risk procedures of adolescent girls who were victims of dating violence. Adolescence 2003; 38: 1-14

6 Reynolds M, Hinchliffe K, Asamoah V, Kouimtsidis C. Trauma and posttraumatic stress disorder in a drug treatment community service. Psychiatrist 2011; 35: 256-60.

7 Reynolds M, Mezey G, Chapman M, Wheeler M, Drummond C, Baldacchino A. Co-morbid post-traumatic stress disorder in a substance misusing clinical population. Drug Alcohol Depend 2005; 77: 251-8.

8 Loose R. The Subject of Addiction: Psychoanalysis and the Administration of Enjoyment. Karnac Books, 2002.
9 Stewart $\mathrm{SH}$, Pihl RO, Conrod $\mathrm{P}$, Dongier M. Functional association among trauma, PTSD and substance-related disorders. Addict Behav 1998; 23: 797-812

10 Woody GE, O'Brien CP, Rickels K. Depression and anxiety in heroin addicts: a placebo-controlled study of doxepin in combination with methadone. Am J Psychiatry 1975; 132: 447-50.

11 Khantzian EJ, Gawin F, Kleber HD, Riordan CE. Methylphenidate treatment of cocaine dependent - a preliminary report. J Subst Abuse Treat 1984; 1: 107-12.

12 Khantzian EJ. The self-medication hypothesis of addictive disorders: focus on heroin and cocaine dependence. Am J Psychiatry 1985; 142 $1259-64$.

13 Khantzian EJ. Treating Addiction as a Human Process: A Plea for a Measure of Marginality. Paper presented at the American Academy of Addiction Psychiatry 11th Annual Meeting and Symposium, Phoenix, 2000 (http://www.addictioninfo.org/articles/250/1/TreatingAddiction-as-a-Human-Process/Page1.html).

14 Milkman $H$, Frosch WA. On the preferential abuse of heroin and amphetamine. J Nerv Men Dis 1973; 156: 242-8.

15 Wurmser L. Psychoanalytic consideration of the etiology of compulsive drug use. J Am Psychoanal Assoc 1974; 22: 820-43.

16 McFall ME, Mackay PW, Donovan DM. Combat-related post traumatic stress disorder and severity of substance abuse in Vietnam veterans. J Studies on Alcohol 1992; 53: 357-63.

17 Brewin CR, Hunter E, Carroll F, Tata P. Intrusive memories in depression. Psychol Med 1996; 26: 1271-6.

18 Kuyken W, Brewin CR. Intrusive memories of childhood abuse during depressive episodes. Behav Res Ther 1994; 32: 525-8.

19 Reynolds $M$, Brewin CR. Intrusive memories in depression and posttraumatic stress disorder. Behav Res Ther 1999; 37: 201-15.

20 McLellan AT, Alterman Al, Cacciola J, Metzger D, O'Brien CP. The fifth edition of the Addiction Severity Index. J Subst Abuse Treat 1992; 180 101-10.

21 Green B. Trauma History Questionnaire. In Measurement of Stress, Trauma and Adaptation (ed. BH Stamm): 366-9. Sidran Press, 1993.

22 Foa EB, Riggs DS, Dancu CV, Rothbaum BO. Reliability and validity of a brief instrument for assessing post-traumatic stress disorder. J Trauma Stress 1993; 6: 459-73.

23 American Psychiatric Association. Diagnostic and Statistical Manual of Mental Disorders, Fourth Edition, Text Revision (DSM-IV-TR). APA, 2000

24 Derogatis LR, Melisaratos N. The Brief Symptom Inventory: an introductory report. Psychol Med 1975; 13: 595-605.

25 Mills KL. 'Between pain and nothing, I choose nothing': trauma, posttraumatic stress disorder and substance use. Addiction 2009; 104: 1607-9 